\title{
LA GOBERNANZA HÍDRICA COMO UN DESAFÍO NACIONAL. EL AGUA EN EL CONTEXTO POST- PANDEMIA. EL CASO DE LERMA, ESTADO DE MÉXICO, MÉXICO'
}

\author{
WATER GOVERNANCE AS A NATIONAL CHALLENGE. WATER IN THE POST- \\ PANDEMIC CONTEXT. THE CASE OF LERMA, STATE OF MEXICO, MEXICO
}

\footnotetext{
Abigail Martínez-Mendoza ${ }^{1}$ * (D); Eloísa Domínguez-Mariani ${ }^{2}$ (D); Mijael Altamirano-Santiago ${ }^{3}$ (D).

1. Universidad Autónoma Metropolitana, México. a.martinez@correo.ler.uam.mx.

2. Universidad Autónoma Metropolitana, México. e.dominguez@correo.ler.uam.mx.

3. Centro de Investigaciones Económicas, Administrativas y Sociales del Instituto Politécnico Nacional, México. maltamiranos@ipn.mx
}

*Correspondencia del autor: Abigail Martínez-Mendoza, email: a.martinez@correo.ler.uam.mx

\section{RESUMEN}

En el contexto de la pandemia por SARS COV-2, una débil gobernanza del agua es un problema para la salud dado que, de manera generalizada escasea; y para este caso, no es apta para el consumo humano y los actores sociales, lo desconocen. El texto analiza y vincula los resultados de dos investigaciones de campos de conocimiento diferentes, pero complementarios respecto a la problemática de la cuenca alta del río Lerma, especialmente el agua subterránea, mostrando un ejercicio interdisciplinario. Los resultados revelan que los actores no identifican los problemas de la cuenca, lo cual limita su integración a los cuerpos colegiados de consejos, comisiones o comités de cuenca, a pesar de que los marcos normativos cuentan con los espacios para éstos. Las OSC, como actores sociales, aún presentan áreas de oportunidad para su incidencia, pese a que en México, hay un largo historial respecto a su participación social.

Palabras clave: gobernanza hídrica; agua subterránea; cuenca; río; actores sociales.

$1 \quad$ El estudio se deriva de dos proyectos: 1) Proyecto de Investigación Institucional "Políticas públicas y perspectiva territorial: caracterización del ciclo hidrosocial en el espacio comprendido por la subcuenca del río Lerma". Acuerdo 104.12, Sesión 104 de Consejo Divisional, celebrada el 3 de marzo de 2020 de la UAM-Lerma; 2) Evaluación de la hidrogeoquímica de humedales naturales y su asociación con factores antropogénicos y naturales, casos Ciénegas de Lerma y del Lago Tláhuac-Xico, Estado de México, México, de la Convocatoria EDOMEX-FICDTEM-2021-01.

Cómo citar:

Martínez-Mendoza, Abigail; Domínguez-Mariani, Eloísa; Altamirano-Santiago, Mijael. (2021). La gobernanza hídrica como un desafío nacional. El agua en el contexto post-pandemia. El caso de lerma, Estado de México, México. Revista de Investigaciones Universidad del Quindio, 33(2), 174-186. https://doi.org/10.33975/riuq.vol33n2.768

Información del artículo: Recibido: 6 mayo 2021; Aceptado: 23 agosto 2021

Revista de Investigaciones Universidad del Quindío, 33(2), 174-186; 2021.

ISSN: 1794-631X e-ISSN: 2500-5782

Esta obra está bajo una licencia Creative Commons AtribuciónNoComercial-SinDerivadas 4.0 Internacional. 


\section{ABSTRACT}

In the context of the SARS COV-2 pandemic, a weak water governance is a health problem given that it is generally scarce; and in this case, it is not suitable for human consumption and the social actors are unaware of it. The text analyzes and links the results of two investigations from different but complementary fields of knowledge regarding the problem of the upper Lerma River basin, especially groundwater, showing an interdisciplinary exercise. The results reveal that the actors do not identify the problems of the basin, this limits their integration to the collegiate bodies of councils, commissions, or basin committees, even though the regulatory frameworks have spaces for these. CSOs, as social actors, still present areas of opportunity for their advocacy, even though in Mexico, there is a long history regarding their social participation.

Keywords: water governance; groundwater; basin; river; social actors.

\section{INTRODUCCIÓN}

Toda cuenca representa un territorio que involucra los aspectos sociohídricos y lo relativo a lo político-económico (Martínez y Altamirano, 2021), una cuenca es un territorio limitado por el flujo de agua movido gravitacionalmente, que influye en el clima y las características del paisaje, esto es en los ecosistemas. Los ríos que son el eje articulador de la cuenca, y de los cuales esta toma su nombre, de acuerdo con sus dimensiones y su articulación con otras cuencas vecinas, pueden denominarse microcuencas, o subcuencas En cada una existe una interacción entre el agua superficial y subterránea. De esta forma, se tienen condiciones particulares para los ecosistemas, los cuales a su vez influyen en como las comunidades hacen uso de los bienes naturales. Así, se construyen relaciones entre sociedades y dentro de ellas, y creando condiciones para el bienestar y la salud dentro de la propia sociedad. Los aspectos anteriores son interdependientes, la salud de la cuenca es la salud de las sociedades.

Estos elementos: cuencas, salud-bienestar, medio ambiente y sociedad, constituyen un prisma de la gobernanza de cuencas, de acuerdo con Parkes et al, (2010), lo cual permite relacionar las diferentes facetas de la gobernanza, en particular permite caracterizar el manejo actual de los recursos hídricos, en especialmente representar las prioridades políticas particularmente las relacionadas con el agua, dejando de lado la visión de bienes naturales. La naturaleza ha sido objeto de recursos para su apropiación, extracción, manipulación y transformación, prevaleciendo así visión de beneficios y rentabilidad para el desarrollo y crecimiento económico (Gudynas, 2015; Georgescu-Roegen, 1996).

De acuerdo con Parkes et al, (2010, pág. 697), el vértice de la gobernanza con la saludbienestar se relaciona con espacios y contextos ecológicos, sociales, culturales y políticos, lo que aplica a escala humana que va desde lo individual hasta lo comunitario; los mismos autores mencionan que la salud ha dejado de ser sólo un tema político, de intervención médica o social para extenderse a la esfera ambiental, de la vivienda, y el alojamiento. El vértice que corresponde a la gobernanza de la salud puede relacionarse a su vez con los ecosistemas, animales y otras especies, y es relevante en el caso de enfermedades infeccionas. Otra relación es relacionada con la salud y la sustentabilidad, y trata no solo de mantener los flujos naturales que mantienen ecosistemas y especies icónicas, sino sostener la salud y vida en general. Una relación más tiene que ver con las determinantes sociales de la salud, enfocadas en la interacción entre la salud, la inequidad social, política y social.

El tema de la pandemia y las medidas planteadas 
para su prevención se relacionan con el acceso al agua por parte de la población y especialmente en casa. Durante la pandemia, mundialmente se hizo de conocimiento general que, para mitigar la propagación del coronavirus, y asegurar resultados más allá de la pandemia, el distanciamiento social, la higiene personal, y en especial, el lavado de manos es y será prioritario contar una mejor gestión hídrica. De acuerdo con el Fondo de las Naciones Unidad para la Infancia (UNICEF, 2020), en el marco del Día mundial del lavado de manos, celebrado cada 15 de octubre de cada año: 1) El 40\% de la población mundial, no dispone de instalaciones para el lavado de manos con agua y jabón en casa; 2) Casi tres cuartas partes de la población de los países menos desarrollados carecen de instalaciones básicas para lavarse las manos en el hogar; 3) en los 60 países identificados con mayor riesgo de crisis sanitaria y humanitaria por el virus, 2 de cada 3 personas carecen de instalaciones básicas para el lavado manos con agua y jabón en el hogar.

Sin embargo, la disponibilidad del agua en las viviendas depende de los organismos operadores, los cuales también deben contemplar la conservación de los ecosistemas. De acuerdo a (Watson et al., 2020), existe un grave deterioro de los ecosistemas. Entre ellos, un ecosistema dependiente del agua subterránea es aquel que, para llevar a cabo sus funciones, requiere de un sistema de flujo de agua, principalmente subterránea, por ejemplo, los humedales y las marismas (Domínguez et al., 2021, pág. 27). Este tipo de ecosistemas se encuentran cada vez más amenazados no sólo por las insaciables necesidades antropogénicas, sino por el nulo reconocimiento a la relación entre la hidrología superficial, subterránea y la ecología (Rhode et al., 2017).

Por lo que, en el contexto de la actual pandemia por SARS COV-2, una débil gobernanza del agua representa un problema de altas repercusiones para la salud. El agua es clave para las medidas de higiene requeridas, por lo que su acceso, disponibilidad y calidad son factores clave para la salud. En el presente se analiza que, en la post-pandemia, la gobernanza en sus diferentes vértices es impostergable.

\section{MARCO CONCEPTUAL}

\section{El agua y pandemia en México}

Para el caso mexicano, la gobernanza hídrica, constituye uno de los objetivos prioritarios del actual Plan Nacional Hídrico (PNH), 20202024, implica una reforma al marco jurídico de mayor inclusión en la gestión del agua, así como requiere focalizar inversiones en zonas y grupos de atención prioritaria. Frente a esto, en México, el consumo de agua en hogares durante la pandemia se incrementó en un 40\% (Martínez, 2020), no sólo por permanecer más tiempo en casa, también por las recomendaciones de lavado constante de manos, aunado es esto, el 25\% los hogares la reciben esporádicamente y 7.0\% no reciben agua (Martínez, 2020).

Antes de la crisis por la Covid-19, la Comisión Nacional del Agua (CONAGUA), tenía una reducción de $45 \%$ en su presupuesto (ANEAS, 2020); esto se traduciría en dificultades para su operación. Durante dicha crisis sanitaria, en México la curva pandémica mostró dos picos, en julio de 2020 y enero de 2021 (SánchezTalanquer, 2021), durante ese periodo, de acuerdo con la entonces directora de la Comisión, Blanca Jiménez, hasta mediados de junio del 2020, se habían suministrado cerca de 167 millones de litros de agua potable a 430 mil personas en 22 entidades, de un total 32 en el país, y se realizaron 70 obras del Programa de Agua Potable, Drenaje y Tratamiento (Jiménez, 2020). Es decir, el suministro no iba emparejado con el desarrollo de la pandemia.

Para el 2021, para el Presupuesto de Egresos de la Federación (PEF) se aprobó un incremento del $1.5 \%$ al sector ambiental, del cual, el 79.5\% está destinado a los programas "Gestión integral y sustentable del agua" e "Infraestructura de 
agua potable, alcantarillado y saneamiento" de la CONAGUA (Müller et al., 2021)

A pesar de esta medida, el $22.4 \%$ de las viviendas en México es vulnerable por infraestructura (Armenta, Ortega et. al., 2020). Es decir, ante el coronavirus, la carencia de agua o precariedad de las instalaciones sanitarias incrementa las posibilidades de que un hogar se convierta en un foco de infección. Esto es, las casas en zonas urbanas con algún grado de marginación, ó zonas rurales precarias, o los asentamientos irregulares carecen del flujo constante de agua y la mayoría de las veces también conexión a vías de suministro (Hernández, 2020).

De acuerdo con el Instituto Nacional de Estadística y Geografía (INEGI, 2020), el 77.6\% de las viviendas en México cuenta con agua entubada, $95.5 \%$ con servicios de alcantarillado, $98.1 \%$ con servicio sanitario, $64.4 \%$ con tinaco y $27.5 \%$ con cisterna. Especialmente, se debe considerar que en México existen 2,471 municipios, de los cuales, señalan Armenta y Ortega que únicamente el $13.3 \%$ de estos no son vulnerables, mientras que el $15.26 \%$ es de alta vulnerabilidad, y el $1.6 \%$ es de muy alta vulnerabilidad (Armenta, Ortega et. al., 2020), esto significa que las viviendas son precarias o carecen de servicios de agua potable y drenaje, baja calidad en los materiales de construcción, así como hacinamiento y limitado o nulo acceso a la salud, por lo que las condiciones de marginación en las viviendas las convierten en blanco fácil de contagios. De manera particular, el Índice de Vulnerabilidad en Infraestructura de la Vivienda ante la COVID-19 (IVIV- COVID) (Armenta, Ortega et. al., 2020), indica que la carencia en servicios por falta de agua entubada en la vivienda en México es del 31.3\%.

Para todo lo anterior, es necesario anotar que en México el agua subterránea constituye el 58.4\% del abastecimiento público (CONAGUA, 2018), esto por su buena calidad y abundante caudal, es una importante fuente de abastecimiento para la población pese a los altos costos de su extracción
(Domínguez et. al., 2021). Esto significa que, para hacer frente a la pandemia por coronavirus, México debe atender pronta e integralmente tanto las necesidades de los ecosistemas de agua como las demandas sociales del consumo y abastecimiento hídrico.

Para el caso delEstado de México, además de vivir la pandemia y el incremento de la demandada el encargado de la Vocalía de la Comisión Agua del Estado de México (CAEM) menciona: la demanda de agua se ha multiplicado por cuatro, si la llave se tenía abierta por cuatro segundos, ahora son 20, es el mismo lavado, y ni decir que ahora nos lavamos más las manos (García, 2020). Aunado a esto, en diciembre de 2020 mientras que en México aún no se declaraban las primeras medidas para enfrentar al coronavirus, fue aprobado un incremento diferenciado a las tarifas de agua para veinte municipios mexiquenses, de los cuales, Amecameca, Cuautitlán Izcalli, Lerma y Toluca tendrían un aumento hasta por $4.6 \%$; mientras que Acolman, Atizapán de Zaragoza, Atlacomulco, Coacalco, Cuautitlán, El Oro, Huixquilucan, Jilotepec, Metepec, Naucalpan, Tecámac, Tepotzotlán, Tlalnepantla, Tultitlán, Valle de Bravo y Zinacantepec se ajustarían según la Unidad de Medida y Actualización (UMA) (Huerta, 2020).

Concretamente, para el municipio de Lerma, el promedio del alza fue del 2\% (Huerta, 2020), y de acuerdo con el Organismo Público Descentralizado de Agua Potable, Alcantarillado y Saneamiento(OPDAPAS), sebuscaincrementar el padrón de usuarios, mejorar y ampliar la calidad del servicio, captación, conducción, distribución, alejamiento y tratamiento de las aguas, así como dar mantenimiento a la red de agua potable y drenaje, capacitar al personal administrativo y técnico, sustituir medidores de agua potable, y un mejorar el equilibrio financiero correspondiente a 14 mil 187 tomas de agua y 12 mil 769 descargas de drenaje con servicio a 56 mil 748 habitantes, así lo señaló Huerta (2020).

En síntesis, la crisis sanitaria por coronavirus 
se ha vivido con dificultades hídricas, tanto el suministro, la disponibilidad, como las tensiones derivadas de los presupuestos asignados a los organismos encargados, la marginación por infraestructura hídrica en los hogares, y el incremento de las cuotas por el servicio. Esto revela una limitada política hídrica.

En un futuro post-pandemia la perspectiva es una gobernanza hídrica urgente. Significa que los gobiernos deberán emprender y reforzar acciones encaminadas a contrarrestar los índices de marginación y vulnerabilidad; esto va más allá de la vivienda en si misma, se trata de consolidar el paradigma de la gobernanza en materia hídrica, pues aún se observa que la mayoría de las veces, las políticas se formulan e implementan únicamente por el gobierno (Cruz-Rubio, 2014). Es superar la transición que va de la gobernabilidad a la gobernanza; es decir, generar nuevos mecanismos de participación y corresponsabilidad con miras a su institucionalización; se trata de la colaboración entre organizaciones de la sociedad civil, sector privado, ciudadanía en general y el gobierno (March y Olsen, 1984; Koelbe, 1995; Hall y Taylor, 1998; Camou, 2000 y 2001).

\section{Gobernanza hídrica}

Hoy en día, el contexto sociopolítico exige contemplar la gobernanza en todos los sectores para atender los problemas públicos; en el ámbito medio ambiental, la gobernanza también es necesaria para cambiar la visión de recursos naturales por la de bienes naturales; ir de la oferta de bienes y servicios en entorno al desarrollo de las sociedades, a una postura del bien común y solidario con la naturaleza.

De acuerdo con Aguilar (2019, pág. 34), los problemas ambientales son el "entretejido causal de interrelaciones entre diversas secuencias causales"; esto es que existen variedad de factores y diversidad de actores con objetivos diferentes en distintos campos. Dada la complejidad, es necesaria la gobernanza pues un solo actor, pese a sus capacidades institucionales, de recursos o poderes, tendrá dificultades para hacer frente a las problemáticas medio ambientales, esto considerando que las capacidades para formular y hacer cumplir reglas y políticas en beneficio del público (Fukuyama, 2013) requiere de fortaleza institucional y liderazgo eficaz, "el gobierno [...] no posee, en el grado requerido, las capacidades financieras, cognoscitivas, tecnológicas, organizativas, la confiabilidad política y moral, el liderazgo social que se requieren [...] por lo que necesita de los recursos y capacidades que los sectores privados, sociales e intelectuales independientes (Aguilar, 2019, pág. 34). En este sentido la gobernanza hídrica también debe ser contemplada. Sin embargo, se advierte que no existe una única definición de gobernanza ambiental que de lugar a la gobernanza hídrica.

Para la Comisión de Gobernanza Global, el significado de gobernanza ambiental es un proceso dinámico de intereses heterogéneos encaminados a la "[...] adopción de acciones de cooperación [...] incluye instituciones formales y regímenes que son vinculantes, así como arreglos informales que las instituciones y personas han acordado o consideran que son de su interés" (The Commission on Global Governance, 1995, pág. 44). Se percibe la interacción entre actores públicos y privados, e instituciones en temas comunes; sin embargo, no se observa al territorio como espacio donde tienen lugar las problemáticas ambientales, con lo que se olvida el concepto de cuenca (o subcuenca o microcuenca, según sea el tamaño)..

Por ejemplo, Valverde, a lo anterior describe a la gobernanza ambiental como "[...] el intento de establecer la relación de la gestión del entorno y sus recursos naturales, los medios de vida de las comunidades y ahora, la adaptación al cambio climático, donde el espacio (la cuenca) es el conjunto natural de localizaciones y el territorio un lugar construido social y políticamente" (2015, pág. 162). En esta propuesta, si bien se habla del territorio, es este un área de gestión; es decir, el origen-destino de los cursos de acción 
gubernamentales porque provee escenarios de factibilidad (Martínez y Altamirano, 2020; Lara y Martínez, 2019); sin embargo, esta visión deja fuera la interacción entre actores públicos y privados, así como el papel de las instituciones.

En cambio, para Castro, Hogenboom y Baud (2015, pág. 18), la gobernanza ambiental será la "formulación y refutación de imágenes, diseños y ejecución de los procedimientos y prácticas que configuran el acceso, control y uso de los recursos naturales entre actores diferentes". Estos autores observan que las acciones dirigidas hacia el medio ambiente constituyen un entramado de actores en distintas escalas. En este sentido, vale señalar que los actores sociales se caracterizan porque su espíritu es incidir en su entorno inmediato de manera proponente (Martínez y Morales, 2021) pues están motivados por la libertad, dignidad y los derechos para incidir o resolver los conflictos que trastocan las libertades y la dignidad (Touraine, 1984; 1997; 2010).

Ahora bien, especialmente, la gobernanza hídrica una de las acepciones de mayor aceptación es aquella que señala que "la autoridad establece reglas claras y ofrece espacios de participación a otros actores y sectores en la gestión del agua, donde las decisiones respecto a las políticas públicas son establecidas por consenso con todos los actores que intervienen en dicha gestión del agua” (Domínguez, 2011, pág. 14).

\section{Instrumentos legales e instituciones de la política hídrica}

La política mexicana en materia hídrica se ha consolidado en el ámbito legislativo y programático. Se trata de todo el corpus legal en torno a los bienes hídricos; esto va desde la misma Constitución, hasta las normatividades para cada sector social; pasando por leyes federales, estatales y municipales, y secretarías (Martínez, 2018). Sin embargo, la materia legal no se traduce directamente en cursos de acción, en políticas públicas que contemplen los momentos mínimos del diseño, implementación y evaluación, e incluso, tampoco la materia legal se desdobla directamente en planes programas y proyectos (Aguilar, 1992; Martínez y Morales, 2020).

En este sentido, si bien hoy en día existe una propuesta para una Ley General de Aguas, que deriva de un ejercicio de participación ciudadana, lo cierto es que aún prevalece la Ley de Aguas Nacionales (LAN), y en el octavo artículo, que la Secretaría de Medio Ambiente y Recursos Naturales (SEMARNAT) y la CONAGUA, serán los actores institucionales encargados de las políticas públicas en materia hídrica. Especialmente, lo que respecta a la implementación de las políticas, es la CONAGUA la institución a nivel nacional encargada de "Integrar, formular y proponer al Titular del Poder Ejecutivo Federal, el Programa Nacional Hídrico, actualizarlo y vigilar su cumplimiento" (LAN, 2016, pág. 13). En el marco de la implementación del PNH (2019-2024), se hace explícita la participación de diferentes actores, como organizaciones sociales o consejos de cuenca, el actual Programa es resultado de " 44 foros específicos [...], en adición a 8 foros de consulta [que suman] 3 mil personas a lo largo y ancho del país" (PNH, 2019, pág. 1).

Es preciso recordar que en el ciclo de las políticas públicas la implementación es aquel momento donde se echa a andar aquello que fue planeado en el diseño de una política, en el caso de la política nacional hídrica, esto se observa cuando los objetivos y acciones plasmadas en el PNH se llevan a cabo por los distintos actores involucrados y según las atribuciones y tareas específicas que les correspondan, especialmente en el quinto objetivo del actual Plan que es mejorar las condiciones para la gobernanza del agua a fin de fortalecer la toma de decisiones y combatir la corrupción (PNH, 2019).

Del último punto se desprenden las acciones encaminadas a la gobernanza hídrica, que resumidamente contemplan la participación social como eje para consolidar el objetivo de 
"Mejorar las condiciones para la gobernanza del agua a fin de fortalecer la toma de decisiones y combatir la corrupción" (PNH 2020-2024, pág. 36); pero se percibe que el Plan tiene una limitación importante, las estrategias no se desagregan en acciones específicas indicativas acerca de cómo se logrará el fortalecimiento, la promoción y el fomento deseados, esto es una limitante para evaluar el avance de tales estrategias, dejando vacíos en las formas y acciones necesarias para consolidar la participación de los actores interesados.

En el ámbito estatal y municipal el marco legal que da vida a la política hídrica, se encuentra la Ley del Agua para el Estado de México y Municipios (LAEMM) y, para el caso de estudio, está el Reglamento Interior de Ecología y Medio Ambiente de Lerma de Villada.

Por su parte, el artículo 2 de la LAEMM se puede leer en el objetivo II, III y $\mathrm{V}$, que se aborda lo correspondiente a la búsqueda de integración y participación de los distintos actores. Se observa que para la gestión de los bienes hídricos están contemplados tanto las autoridades correspondientes, como los organismos operadores y los usuarios. Asimismo, en el artículo 11 menciona "La promoción de una participación informada y responsable de las personas, como base para la gestión integral de agua, el manejo sustentable del agua y el fomento a la cultura del agua" (LAEMM, 2013, pág. 11). A pesar de esto, esta ley estatal carece de un señalamiento claro de las formas de participación para las organizaciones sociales y los Consejos de cuenca, o bien, de un reglamento secundario que apunte al respecto.

En cuanto, al Reglamento Interior de Ecología y Medio Ambiente (RIECMA) del municipio de Lerma de Villada, en general están las acciones que competen al ayuntamiento respecto a la prevención de la contaminación, especialmente el capítulo cuarto relativo a la protección y aprovechamiento del agua, el mal uso, las aguas residuales y la calidad del agua en el municipio.
Sin embargo, en este nivel de legislación, no se logra observar lo relativo a la gobernanza hídrica, ya que destaca la tarea de un solo actor.

\section{MATERIAL Y MÉTODOS}

Este trabajo se desarrolla desde la perspectiva interdisciplinaria, toda vez que el problema se aborda desde el ámbito de las ciencias sociales y las ciencias básicas, especialmente desde las políticas públicas y la hidrogeología. Hace uso de la investigación cualitativa y cuantitativa, por el uso de teorías y métodos que habrán de ser soporte para el análisis y el tratamiento de la información. Particularmente, se empleó la investigación documental para recolectar, organizar, analizar información y datos tanto de instancias gubernamentales como de organizaciones de la sociedad civil, leyes y otros instrumentos normativos, y bases de datos; todo relativo al tema del agua.

En especial se emplea información relevante y vinculada de trabajos de investigación que las autoras asesoraron, y observan una relación que responde a la delimitación temática, territorial y conceptual, abordada desde métodos de investigación diferentes. El primer trabajo, en el marco de las políticas públicas, da cuenta de las organizaciones de la sociedad civil inscritas en la subcuenca metropolitana del río Lerma, y cuyo objetivo fue determinar las destrezas y capacidades de las Organizaciones de la Sociedad Civil (OSC) para el ejercicio de la gobernanza hídrica. La investigación de Cruz (2018) identificó territorialmente y contactó a las organizaciones adscritas sobre la cuenca y cuyas acciones estuvieran vinculadas al río o la cuenca alta; es decir, empleó la investigación cualitativa, documental y de trabajo campo con entrevistas semiestructuradas.

El segundo trabajo, que deviene de la hidrogeología, ofrece un diagnóstico de las aguas subterráneas de la misma cuenca, específicamente aborda el comportamiento hidrogeoquímico, a partir de la observación 
que hace CONAGUA (2018) con relación a la extracción de agua subterránea para el consumo de la población. El trabajo de Berrón (2020) tomó muestras de norias, pozos y del mismo río Lerma en la cuenca alta, especialmente en el tramo que atraviesa el municipio del mismo nombre; en suma, esta investigación es de tipo cuantitativa y de campo a partir de la recolección de muestras de aguas subterráneas.

El vínculo entre estas las dos investigaciones es en torno a la gobernanza hídrica para el caso de Lerma, partir de considerar que para la gobernanza se requiere de la participación de diferentes actores, a su vez, estos deben contar información actualizada respecto a las condiciones sobre las que se pretenden dirigir las acciones, pues contar con información es parte de las habilidades para involucrase en el diseño, implementación o evaluación de políticas públicas, y en todo caso, en planes, programas o proyectos (Martínez y Morales, 2021). Especialmente, dicha relación interdisciplinaria permite dar cuenta del desafío para hacer frente a la pandemia.

\section{RESULTADOS}

Tanto Berrón (2020) como Cruz (2018) observan una dinámica constante, diferentes actores vinculados al tema de aguas en la Cuenca Alta del río Lerma (CARL), pero encapsulados en sus propias dinámicas; esto es que, la interacción entre ellos es mínima o bien, no forman parte del espectro de actores que harían posible la gobernanza hídrica para río y la cuenca del alto Lerma. Cabe decir que, en el Estado de México, entidad federativa en donde se ubica la cuenca, tiene con más de 3,142 OSC registradas (Cruz, 2018, pág. 32); y el trabajo de ambas autoras, entre 2018 y 2020, reporta cuatro organizaciones, de las cuales una se denomina colectivo, y tres más fundaciones.

Al respecto, Berrón (2020) reporta una fundación conformada en enero de 2003, enfocada en la problemática de la contaminación del río Lerma y que busca la participación de colectivos del conocimiento de las culturas originarias, científicos, investigadores, gobiernos federal, estatal y municipal a través de foros, reuniones, donde se abarque la problemática hídrica. Su directora, mencionó sobre la falta de personal e interés por parte del gobierno mexicano por atender las necesidades medioambientales. Respecto a la misma fundación, Cruz (2018) señaló que se creó en 2002, que está conformada por habitantes y avecinados de la CARL, de comunidades indígenas Náhuatl, Otomí y Mestiza; y que en el entonces estudio, no se obtuvo una respuesta para a realizar una entrevista.

En esta misma tesitura, Berrón (2020, pág. 28) identificó un colectivo, del cual señala que su objetivo es promover la protección del ambiente, flora y fauna, del desarrollo sustentable a nivel regional y comunitario de los municipios que conforman la subcuenca del río Lerma. Indica, la misma autora, que participan instituciones como la Universidad Autónoma del Estado de México, el Colegio de Estudios Científicos y Tecnológicos del Estado de México, Universidad Tecnológica del Valle de Toluca, y la Universidad Autónoma Metropolitana, así como la Secretaría de Obra, de Desarrollo Urbano y Metropolitano, la Comisión Nacional de Áreas Protegidas, Colegio de Ingenieros del Estado de México, y los Ayuntamientos de Toluca, Lerma, Capulhuac, Ocoyacac, Temoaya, Santiago Tianguistenco , Xalatlaco, Texcaliacac, Almoloya del Río, Santa Cruz Atizapán, Xonacatlán, Otzolotepec, y Temoaya.

De lo anterior, Cruz (citado en Berrón, 2020, pág. 29) señala: “[...] que estas organizaciones, aunque están presentes no cuentan con un papel representativo para ayudar a la problemática hídrica debido a que no forman parte del consejo de la subcuenca, limitándolos así a no tener una participación para implementar medidas". Al respecto, es posible decir, que ambas autoras coinciden en el señalamiento.

Cruz (2018) describió tres organizaciones que 
expresamente señalaron trabajar con temas vinculados al río y a la CARL, y se denominan fundaciones, una de ellas coincide con la que Berrón contactó en 2020. El estudio de Cruz (2018) indica que las tres OSC presentan debilidades en las capacidades, destrezas técnicas y/o políticas, así como de arraigo local para formar y tomar parte de las decisiones, planes, programas y proyectos.

En resumen, Cruz (2018) señala que las capacidades organizacionales serán las habilidades de la organización para el funcionamiento sostenible, eficaz, estable y sólido; las destrezas técnicas serán los conocimientos que permitan generar propuestas viables y ejecutables; y, las destrezas políticas se observarán en las relaciones con otras organizaciones o autoridades para lograr insertarse en la arena política; mientras que el arraigo social es la habilidad de las OSC para vincularse con otros demás actores, generar y ejecutar propuestas. Para el caso que aquí se pretende analizar y vincular, se retoma lo referente a destrezas técnicas, las cuales implican el contar con información.

Tabla 1: Destrezas técnicas de las OSC en la CARL

\begin{tabular}{|c|c|c|c|c|}
\hline OSC & & $\begin{array}{c}\text { Fundación Vive Río } \\
\text { Lerma Guanajuato } \\
\text { Chapala S.C. }\end{array}$ & Fundación Tláoc A.C, & $\begin{array}{l}\text { Fundación Comunidades del Alto } \\
\text { Lerma A.C (también estudiada por } \\
\text { Berrón 2020) }\end{array}$ \\
\hline $\begin{array}{l}\text { Destrezas } \\
\text { técnicas }\end{array}$ & $\bullet$ & $\begin{array}{l}\text { Reconocen los } \\
\text { problemas de la } \\
\text { cuenca. } \\
\text { No mencionaron } \\
\text { metas y/u objetivos } \\
\text { que hayan diseñado } \\
\text { de forma clara. } \\
\text { Han trabajado } \\
\text { con gobiernos } \\
\text { municipales y cuentan } \\
\text { con el Registro } \\
\text { Federal de las OSC. } \\
\text { Identifican algunos } \\
\text { procedimientos } \\
\text { gubernamentales. }\end{array}$ & 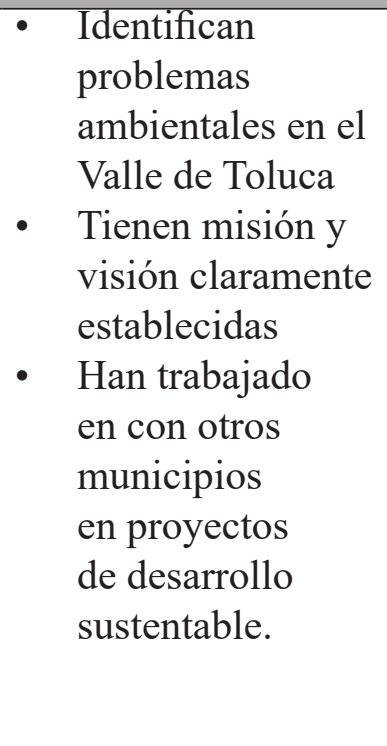 & $\begin{array}{l}\text { - Reconocen las carencias en } \\
\text { materia de atención a los } \\
\text { humedales de la cuenca del río } \\
\text { Lerma } \\
\text { - No tienen misión y visión } \\
\text { - La organización surgió de la } \\
\text { motivación de líderes que habitan } \\
\text { en la zona } \\
\text { - establecidos. } \\
\text { Es difícil tener información } \\
\text { sobre la organización y sus } \\
\text { actividades }\end{array}$ \\
\hline
\end{tabular}

Fuente: Elaboración propia con base en Cruz (2018).

Se pueden observar que ninguno de los casos ahondó en los problemas de la CARL y del río Lerma. En este sentido, es que el trabajo de Berrón (2020) cobra importancia, pues identifica elementos que aportan conocimientos básicos que permitan generar propuestas viables, es decir, destrezas técnicas, tanto para las organizaciones como para el gobierno:

- El sistema acuífero del valle de Toluca que se encuentra en la CARL se compone de una porción en rocas volcánicas fracturadas en la Sierra de las Cruces (límite con la Zona Metropolitana de la Ciudad de México); otra porción que corresponde a un acuífero de materiales granulares-volcánicos, bajo los sedimentos lacustres. En estos se ubican los pozos de mayor productividad y calidad para el suministro de agua potable. Sobre los sedimentos lacustres se han perforado norias por parte de las comunidades, las que son cada vez más escasas.

- El municipio de Lerma se abastece de pozos profundos uso público urbano con $59.92 \%$, 
seguido del industrial $14.42 \%$, diferentes usos con $16.82 \%$, servicios $7.3 \%$ y agrícola con $1.48 \%$.

- Las comunidades ubicadas en la zona alta (Sierra de las Cruces), obtienen principalmente agua de manantiales; y los habitantes de la cabecera municipal, zonas residenciales y en la zona industrial, se abastecen de los pozos profundos.

- Las norias (2mts. de profundidad), y que son utilizadas por poblaciones conectadas o no a la red de abastecimiento, no cumplen la Norma Mexicana (NOM-127) para parámetros como turbidez, alcalinidad, oxígeno disuelto, dureza, color y nitrato, por lo que el agua no se recomienda para consumo humano.

- La concentración de oxígeno disuelto obtenido fue de $0 \mathrm{mg} / \mathrm{l}$, en la mayoría de las muestras, estando por debajo de los criterios de calidad del agua.

- Los valores de turbidez en las muestras de río sobrepasan los niveles de un máximo de 5 UTN, resultado del alto contenido de sólidos suspendidos debido a las descargas de aguas residuales.

- Hay altas de nitratos en el agua, por lo que se podría considerar que existe infiltración de agua de riego con alto contenido de fertilizantes, o de aguas residuales.

Por otra parte, tanto Berrón (2020) como Cruz (2018) señalan que estas organizaciones no participan en la toma de decisiones sobre el territorio de estudio: "Respecto ala representación de [la] sociedad civil, el subdirector técnico de la Comisión de Cuenca del río Lerma (...) señala que, si bien algunas organizaciones han trabajado con ellos, no existe como tal una participación de éstas, pues solamente los presidentes municipales toman las decisiones" (Cruz, 2018, pág. 76).

De acuerdo con la CONAGUA (2016), en México existen de 26 consejos de cuenca, y 215 órganos auxiliares, de los cuales 36 son comisiones de cuenca a nivel de subcuenca, entre las cuales está la Comisión de Cuenca del río Lerma. El reglamento interno de la Comisión de Cuenca del río Lerma (RICCRL, 2017), se lee que serán atribuciones del coordinador general de la Comisión:

Impulsar la participación de organizaciones públicas, privadas y sociales en la ejecución de programas y proyectos para la recuperación de la Cuenca del Río Lerma (Art. 6º, RICCRL, 2017, pág. 3).

Lo anterior está alineado con los señalamientos de la LAN (2016) sobre la integración mixta y colegiada de los consejos, comisiones y comités de cuenca; sin embargo, el RICCRL (2017) no se indica si las organizaciones tendrán un papel activo, con voz y voto. En este sentido, bien señala Cruz (2018), los consejos de cuenca son relevante para formular, modificar, seguir, implantar o evaluar la programación hídrica, y además de eso, hacer cumplir lo que la Ley indica:

[Los consejos de cuenca] señalarán los mecanismos de consulta que aseguren la participación y corresponsabilidad en el desarrollo de actividades, de los usuarios y demás grupos sociales interesados (LAN, 2016, pág. 38).

De todo lo anterior, es relevante anotar que los actores sociales, especialmente las OSC colaborando con los gobiernos, dan paso a un esquema gobernanza. En México, las OSC tienen un largo historial: 1) tras el sismo del 19 de septiembre de 1985, dio paso a la participación voluntaria y organizada; 2) En 1993, se constituyó el Fondo de Coinversión Social que apoyó a las OSC con recursos a través de concursos; 3) En 1994, se formó el Consejo Nacional de Organizaciones No Gubernamentales y los Consejos Técnicos de Organizaciones no Gubernamentales (CTONG), para brindar asesoría; 4) Para el año 2000, el Fondo de Coinversión Social cambió a Programa de Coinversión Social pero continúo capacitando y otorgando recursos para las Organizaciones; 5) En 2004, se publicó la Ley Federal de Fomento a las Actividades Realizadas por Organizaciones de la Sociedad Civil (Martínez, Altamirano y 
Cruz, 2021; Martínez y Morales, 2021).

Actualmente, la administración federal que gobierna desde 2018 hasta 2024, mantiene abiertamente un distanciamiento con las OSC, esto es que aquella colaboración entre las organizaciones y los gobiernos, y que daba paso a la construcción de una gobernanza, se ha frenado.

\section{CONCLUSIONES}

La gobernanza hídrica en un contexto de postpandemia es urgente, toda vez que las aguas subterráneas aportan casi un $60 \%$ para el consumo humano en México. Las aguas que no se ven, son las menos atendidas y entorno a ellas, se generan mitos en cuanto a su presencia y renovabilidad; sin embargo, es imposible separarlas del ciclo hidrológico del cual todos resultan beneficiados.

También es impostergable la gobernanza porque los actores, como los Consejos de cuenca y las OSC no trabajan conjuntamente. Por un lado, los consejos no incorporan a las organizaciones, cometiendo con esto, una falta a la ley; por otra parte, las OSC, presentan carencias técnicas, sobre todo aquellas vinculadas con el conocimiento y origen de las problemáticas específicas, especialmente no incorporar para su operación del concepto de cuenca. En suma, es básico conocer, por un lado, si el agua es apta para el consumo, y por otro, la sustentabilidad en su aprovechamiento, ambos aspectos tan necesarios para los tiempos que ahora vivimos de manera generalizada.

De este modo, es difícil hablar de gobernanza hídrica para la CARL. En efecto, Berrón (2020), con su estudio con énfasis cuantitativo, confirma el trabajo cualitativo de Cruz (2018). En suma, se puede decir que las organizaciones en la CARL carecen de destrezas técnicas, y por parte de la administración en tres niveles no se tiene instrumentación para la obtención de datos puntuales o bien, no es de carácter abierto. Por lo que, a pesar de las motivaciones por coadyuvar con los problemas cuenca y su río, lo cierto, es que la carencia de conocimientos puntuales limita las rutas de acción que puedan emprender; esto es, se carece de un mapa de ruta que nos guie por la senda del pandemia y post-pandemia.

\section{REFERENCIAS}

1. Aguilar, L. F. (2019). "Problemas y propuestas desde la gobernanza ambiental un estudio introductorio" en Rosete, F., Escalera, C., Ayala, B., García-Frapolli, E., y Galán, C. (Coords.) El Ciclo De Políticas Públicas Casos Selectos De La Política Ambiental Mexicana Para La Enseñanza. UNAM, México.

2. Aguilar, L. F. (1992). La hechura de las políticas, México: Miguel Ángel Porrúa.

3. Ortega, A., Armenta, C., García, H. A.,. García, J. R. (2020), Índice de vulnerabilidad en infraestructura de la vivienda ante el COVID-19 (IVIV-COVID). Centro CEMEX-TEC, México. Disponible en: https://www.cdcs.com.mx/public/docs/covid_docs/es/enpdf-REPORTE_ ESPANOL_Tecnica_IVIV-COVID_Oficial.pdf.

4. Asociación Nacional de Empresas de Agua y Saneamiento de México (ANEAS) (2020). "Retos de los organismos operadores ante el Covid-19", en Agua y saneamiento, 87, junio. Disponible en: https://aneas.com.mx/wp-content/uploads/2020/07/AyS87fin.pdf.

5. Berrón, Y. (2020). "Propuesta de gobernanza del agua subterránea para el municipio de Lerma, Estado de México". Tesis. Licenciatura en Universidad Autónoma Metropolitana.

6. Camou, A. (2000). "La múltiple (in)gobernabilidad: elementos para un análisis conceptual", en Revista Mexicana de Sociología, 62 (4):159-188.

7. Camou, A. (2001). "Estudio preliminar", en Camou, A. (comp.) Los desafíos de la gobernabilidad. Estudio preliminar y compilación, México: FLACSO/IIS-UNAM/Plaza y Valdés.

8. Castelán, E. (2001). Los consejos de cuenta en México. Centro del Tercer Mundo para el Manejo del Agua, A. C., México. 
9. Castro, F., Hogenboom, B., y Baud, M. (2015), Gobernanza ambiental en América Latina, CLACSO-EGOV. Ciudad Autónoma de Buenos Aires.

10. Comisión de la Cuenca del Río Lerma. (2016). Recuperado de: http://cuencalerma.edomex.gob. $\mathrm{mx} /$.

11. Comisión Nacional del Agua. (CONAGUA). (2016). Consejos de Cuenca, Recuperado de: https:// www.gob.mx/conagua/documentos/consejos-de-cuenca.

12. Comisión Nacional del Agua. (CONAGUA). (2018). Estadísticas del agua en México, 2018. Disponible en: http://sina.conagua.gob.mx/publicaciones/EAM_2018.pdf.

13. Commission on Global Gobernance. (1995). Our Global Neighborhood: The Report of the Commission on Global Governance. Oxford: Oxford University Press.

14. Cruz, G. (2018). "El papel de las organizaciones de la sociedad civil en la implementación del programa nacional hídrico 2014-2018: el caso de la subcuenca metropolitana de la cuenca del río Lerma". Tesis. Licenciatura en Universidad Autónoma Metropolitana. México: UAM.

15. Cruz-Rubio, C. (2014). Hacia el gobierno abierto: Una caja de herramientas. OEA. Serie: OEA/ Ser.D/XX SG/SAP/.

16. Domínguez, J. (2011). Hacia una buena gobernanza para la Gestión Integrada de los Recursos Hídricos. Documento Temático de Las Américas. Grupo de Buena Gobernanza, 62.

17. Domínguez, M. E., Vargas, C. C., Sandoval, G. J., y Martínez, M. A. (2021). "Extracción intensiva de agua subterránea y la supervivencia de las ciénegas de Lerma, estado de México". Impluvium, 7(15), abril-junio. Disponible en: http://www.agua.unam.mx/assets/pdfs/impluvium/numero15. pdf.

18. Fukuyama, F. (2013), “What Is Governance?”, en Governance, 26:347-368. Disponible en: https:// doi.org/10.1111/gove.12035.

19. García, A. (6 de julio de 2020). "Aumenta consumo de agua en municipios metropolitanos por medidas sanitarias", en El sol de Toluca. Disponible en https://www.elsoldetoluca.com.mx/local/ aumenta-consumo-de-agua-en-municipios-metropolitanos-por-medidas-sanitarias-5456101.html.

20. Georgescu, N. (1996). La ley de la entropía y el proceso económico. Fundación Argentina. Disponible en: http://fcmanrique.org/fcm-publicacion/la-ley-de-la-entropia-y-el-procesoeconomico-2/?lang=es.

21. Gudynas, E. (2015). Derechos de la naturaleza: Ética Biocéntrica y Políticas ambientales. Tinta Limón.

22. Hall A., P., y Taylor C. R. (1998). "The potential of historical institutionalism a response to Hay and Wincott", en Political Science, 46(5), USA.

23. Hernández, S. M. (2020). "El derecho a la vivienda adecuada para la época de la COVID-19 y después: precisiones conceptuales e implicaciones para los grupos históricamente discriminados", en Vivienda Infonavit. México Disponible en: https:/infonavit.janium.net/janium/ Documentos/78175.pdf.

24. Huerta, V. (8 de diciembre de 2020). "Aprueban tarifas de agua con alzas máximas del 4.6\%; Toluca y Lerma incluidos", en El sol de Toluca. Disponible en https:/www.elsoldetoluca.com.mx/ local/aprueban-tarifas-de-agua-con-alzas-maximas-del-4.6-toluca-y-lerma-incluidos-6109919. html.

25. Instituto Nacional de Estadística y Geografía (INEGI) (2020). XII Censo General de Población y Vivienda 2000. México: INEGI. Disponible en: https://www.inegi.org.mx/programas/ccpv/2000/.

26. Jiménez, B. (2020). "Comunidades vulnerables, prioridad de la Conagua”, en Agua y Saneamiento, 87, Junio. Disponible en: https://aneas.com.mx/wp-content/uploads/2020/07/AyS87fin.pdf.

27. Koelbe, A. T. (1995), "The new institutionalism in political science and sociological", en Comparative Politics, 29(2).

28. Lara, M. y Martínez, J. (2019). "Focalización de la población potencial en las políticas públicas: un reto para el análisis territorial". En De la Rosa, J. J., y García, A. P. (coords). Políticas Publicas con respectiva territorial. México: Juan Pablos Editores-Universidad Autónoma Metropolitana.

29. Ley del Agua para el Estado de México y Municipios. (15 de febrero del 2013). Decreto Número 52, Periódico Oficial Gaceta del Gobierno,.

30. March, J. G. y Olsen, J. P. (1984). "The new institutionalism: organizational factor in political life", en American Political Science Review, 78 (3):734-749.

31. Martínez, A; Altamirano, M., y Cruz, H. (2021). "Actores sociales en la subcuenca del río Lerma en México. Insuficiencia de habilidades para enfrentar el problema público del agua”, en Revista 
Inclusiones Vol: 8 num Especial, 01-14. Disponible en: https://revistainclusiones.org/index.php/ inclu/article/view/1092.

32. Martínez, A. (2018) "Valles Centrales. La Confrontación De Instrumentos De Política Hídrica, Derechos Y Resoluciones", en Ramos A., Méndez, B. Propuestas de política en regiones y municipios en Oaxaca. AM Editores.

33. Martínez, A., y Altamirano, M. (2020). "La política hídrica con perspectiva territorial: Valles Centrales de Oaxaca", en Investigación Administrativa, 49(126). México: Instituto Politécnico Nacional, Disponible en: http://www.redalyc.org/articulo.oa?id=456063405008.

34. Martínez, A., y Morales, G. M. (2021). "Adecuaciones de políticas sociales transexenales para armonizar con la 4t: el caso del programa de coinversión social”, en Sosa, J. M., Hernández, M. R, Dávila, J. M. (coords). Las políticas y los programas públicos en el marco del gobierno de la 4T en México ¿Continuidad o Transformación?. México: Universidad Autónoma Metropolitana, ITACA.

35. Martínez, P. (24 de abril de 2020) "El consumo de agua en hogares ha aumentado $40 \%$ durante la pandemia, pero no todos reciben el líquido", en El economista, Disponible en: https://www. eleconomista.com.mx/arteseideas/El-consumo-de-agua-en-hogares-ha-aumentado-40-durante-lapandemia-pero-no-todos-reciben-el-liquido-20200424-0063.html.

36. Müller, A. Niño, G. Alcerreca, W. y Mendoza, J. (2021). Análisis del presupuesto destinado a medio ambiente y cambio climático en el PEF 2021. México: IKI Alliance. Disponible en: https:// iki-alliance.mx/analisis-del-presupuesto-destinado-a-medio-ambiente-y-cambio-climatico-en-elpef-2021/.

37. Norma Oficial Mexicana NOM-127-SSA1-1994, Salud ambiental. Agua para uso y consumo humano. Límites permisibles de calidad y tratamientos a que debe someterse el agua para su potabilización. Disponible en: https://www.pediatria.gob.mx/archivos/burbuja/13.4_NOM-127SSA1-1994_Salud_Ambiental_Agua_limites_permisibles_de_calidad.pdf.

38. Parkes, M. W., Morrison, K. E., Bunch, M. J., Hallström, L. K., Neudoerffer, R. C., Venema, H. D., \& Waltner-Toews, D. (2010). "Towards integrated governance for water, health and socialecological systems: The watershed governance prism". Global Environmental Change, 20(4): 693704. Disponible en: https://www.sciencedirect.com/science/article/pii/S0959378010000579.

39. Plan Nacional Hídrico (PNH) 2020-2024. (2019). México, Gobierno Federal, Disponible en: http://pnd.gob.mx.

40. Reglamento Interior de Ecología y Medio Ambiente. (2016). Prontuario de Reglamentación Interna Municipal 2016-2018. Disponible en: http://www.lerma.gob.mx/wp-content/uploads/docs-pages/ PRONTUARIO_REGLAMENTOS.pdf.

41. Rhode, M. M., Froend, R., y Howard, J. (2017). "A Global Synthesis of Managing Groundwater Dependent Ecosystems Under Sustainable Groundwater Policy”. Groundwater, 55(5):293-301. Disponible en: https://ngwa.onlinelibrary.wiley.com/doi/10.1111/gwat.12511.

42. Sánchez-Talanquer, M., et. al (2021). La respuesta de México al Covid-19: Estudio de caso. San Francisco, EUA.: Instituto de Ciencias de la Salud Global

43. Touraine, A. (1984). Le retour de l'acteur. París: Fayard.

44. Touraine, A. (1997). Pourrons-nous vivre ensemble? Égaux et différents. Paris: Fayard.

45. Touraine, A. (2010). Après la crisis. París: Seuil.

46. UNICEF (15 de octubre de 2020). "La falta de instalaciones para lavarse las manos con jabón expone a millones de personas a un riesgo mayor de contraer la COVID-19 y otras enfermedades infecciosas", en UNICEF,. Disponible en: https://www.unicef.org/lac/press-releases/faltainstalaciones-para-lavarse-las-manos-con-jabon-expone-millones-de-personas.

47. Valverde, G. (2016). "La gobernanza ambiental como enfoque para la cogestión adaptativa", en Integra Educativa, IX(1). Disponible en: http://www.scielo.org.bo/pdf/rieiii/v9n1/v9n1_a10.pdf.

48. Watson, J. E., Keith, D. A., Strassburg, B. B., Venter, O., Williams, B., \& Nicholson, E. (february 20, 2020). "Set a global target for ecosystems". Nature, 578:350-362. 